\title{
Size Effect on ESD Threshold and Degradation Behavior of InP Buried Heterostructure Semiconductor Lasers
}

\author{
Jia-Sheng Huang ${ }^{*}$ and Hanh Lu
}

Emcore, Broadband Division, 2015 W. Chestnut St., Alhambra, CA 91803, USA

\begin{abstract}
Optoelectronic components such as laser diodes and light-emitting diodes are vulnerable to electrostatic discharge (ESD) and electrical overstress (EOS). In this paper, we extensively study the size effect on ESD performance of buried heterostructure (BH) distributed feedback (DFB) InGaAsP/InP lasers. We show that the ESD threshold and degradation behavior of $\mathrm{BH}$ lasers are correlated with the cavity length and contact width. The ESD threshold increases linearly with increasing cavity length and contact width. For the ESD degradation behavior, the occurrence frequency of hard degradation, a behavior characterized with a sudden jump in threshold current during the ESD voltage ramp, decreases with increasing cavity length. We also show that the dielectric layer is influential in ESD performance. The physical mechanisms of the ESD behavior will also be discussed.
\end{abstract}

\section{INTRODUCTION}

ESD continues to be the common cause of reliability failure for microelectronic devices including electronic integrated circuits (ICs) and optoelectronic components. It is prudent for the component manufacturers to incorporate ESD robustness into their device design. Such design-in ESD is especially important as we are evolving into the nano-electronics era, where the increasing number of devices features in the nanometer range is being integrated into each communication system.

ESD damage could result from device handling during assembly and test manufacture, machine transient, etc. For Fabry-Perot (FP) and distributed feedback (DFB) semiconductor lasers, ESD could cause changes in device performance parameters such as threshold current, optical power and optical spectrum. For DFB lasers, ESD damage could cause changes in single mode lasing, leading to side-modesuppression-ratio (SMSR) failure. Additionally, the kink, often associated with the ESD damage, could also adversely affect the device linearity during modulation in the analog applications. For the application such as dense wavelengthdivision-multiplexing (DWDM), the wavelength stability with respect to the specified channel spacing could also be affected by the ESD damage. For example, the specification limit of the wavelength drift for $50 \mathrm{GHz}$ is $0.1 \mathrm{~nm}$, which is very important for the DWDM application of $0.4 \mathrm{~nm}$ channel spacing. Hence, it is critical to ensure that laser components achieve ESD robustness in order to meet the performance requirement of fiber optic system.

The ESD sensitivity classifications have been highlighted by Telcordia technical reference (TR-NWT-000870) [1]. Class 1 is defined as the minimum human-body-model (HBM) ESD failure threshold (Vth) less than $100 \mathrm{~V}$, and it

*Address correspondence to this author at the Emcore, Broadband Division, 2015 W. Chestnut St., Alhambra, CA 91803, USA;

E-mails: jshuang@emcore.com, jshuang6@yahoo.com certainly includes types of small area photodiodes. Class 2 covers the ESD failure threshold from $100 \mathrm{~V}$ to $500 \mathrm{~V}$, typically including metal-on-semiconductor (MOS) integrated circuits (ICs). Class 3 covers the ESD failure threshold between 500V and 2000V, including devices such as well-designed MOS and bipolar ICs. Perhaps for this historical reason, most of the IC and optoelectronic device manufacturers set $500 \mathrm{~V}$ as the ESD requirement. Class 4 covers ESD failure threshold between $2000 \mathrm{~V}$ and $4000 \mathrm{~V}$. Many well-designed and processed semiconductor laser diodes (LD) and light emitting diodes (LED) are expected to fall into this category. Finally, Class 5 contains the ESD failure threshold above 4000V. Typically, passive components such as resistors meet Class 5 criterion.

Besides the ESD classifications, JEDEC also document the standards of ESD qualification, apparatus calibration and waveform verification [2].

Optoelectronic manufacturers and research laboratories have been striving to meet the 500V ESD requirement [3-6]. Some companies have shown that the laser devices exhibit ESD threshold above $2000 \mathrm{~V}$ in reverse polarity [7-11]. Despite of the technological achievements in ESD performance, there was only limited work done previously on the basic understanding of ESD characteristics and physical mechanisms [3-7]. Although the ESD thresholds of FP and DFB lasers were reported, the interplay of ESD and electrical overstress (EOS), the size effect and the ESD degradation behavior, have not been well understood [8-9]. In this paper, we study the ESD degradation characteristics of semiconductor lasers. We show that the ESD threshold and degradation behaviors are dependent upon the size of the chip. We have also shown that the ESD damage threshold was influenced by the dielectric properties, the frequency of ESD pulses and laser device structure. We will discuss the physical mechanism for the size and processing effects.

\section{EXPERIMENTAL SETUP}

An active epitaxial layer consisting of InGaAsP multiquantum wells (MQW) was grown on n-type InP substrate 
using metal organic chemical vapor deposition (MOCVD). To form single-mode DFB lasers, grating layers were grown and defined by holographic technique. In the holographic technique, the grating period was created by the interference of coherent beams. A He-Cd laser of $325 \mathrm{~nm}$ wavelength and a diffraction pinhole were used to produce the coherent beams. For the buried heterostructure $(\mathrm{BH})$ laser (shown in Fig. 1a), the active region was etched to form a mesa structure. Subsequently, $\mathrm{p}$-InP and n-InP blocking layers were grown, followed by $\mathrm{p}$-InP and $\mathrm{p}$-InGaAs layers to minimize leakage. For ridge waveguide (RWG) laser (shown in Fig. 1b), a ridge of $1.8-2.0 \mu \mathrm{m}$ was formed by wet etch to provide current and optical confinement. For p-metal, $\mathrm{Au} / \mathrm{Ti} / \mathrm{Pt} / \mathrm{Au}$ was deposited on the p-InGaAs contact layer and annealed. The wafer was lapped to enable the scribing. Finally, $\mathrm{AuGe} / \mathrm{Ni} / \mathrm{Au}$ was deposited on the backside for $\mathrm{n}$-metal contact. $\mathrm{Cr} / \mathrm{Au}$ was deposited for protection and solder joint. To study the size effect, cavity length was varied from $250 \mu \mathrm{m}$ to $750 \mu \mathrm{m}$, and contact width was varied from $5.3 \mu \mathrm{m}$ to $8.1 \mu \mathrm{m}$.
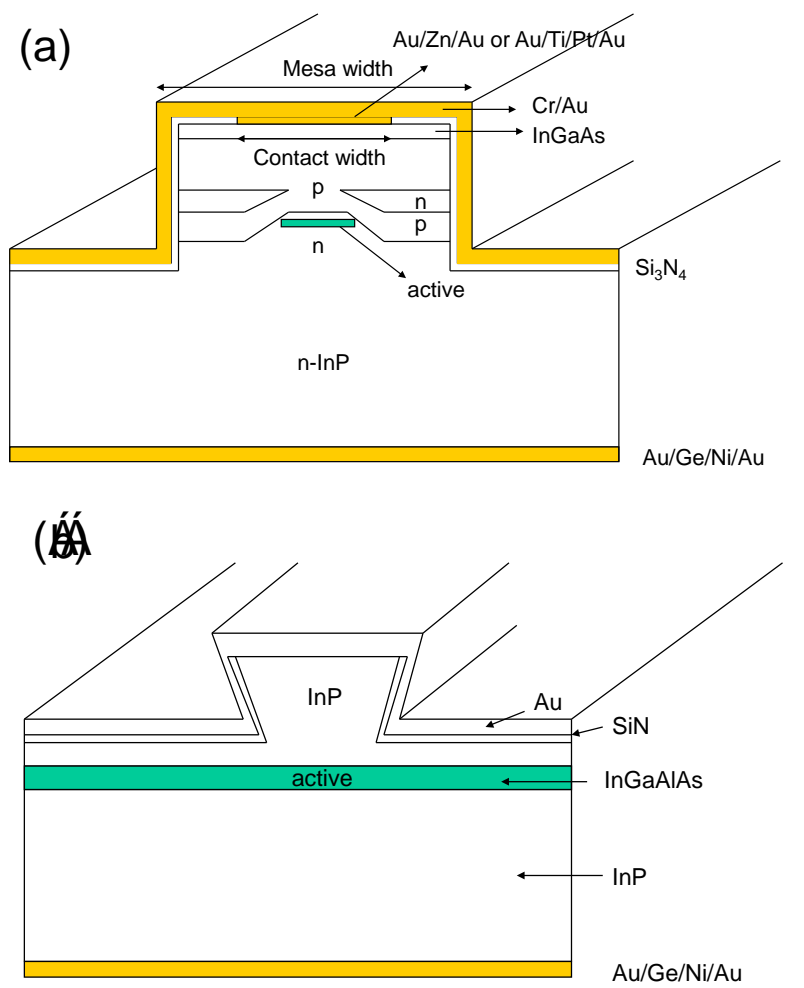

Fig. (1). Schematics of (a) BH laser structure and (b) RWG laser structure.

After completion of wafer processing, wafers were cleaved into bars, and the bars were diced into chips. To facilitate handling and testing, the chips were attached and wire bonded to the ceramic block. The solder joint between the chip and block was AuSn.

Human-body-model (HBM) ESD simulator consisting of a circuit of $1.5 \mathrm{k} \Omega$ and $100 \mathrm{pF}$ was used for the ESD characterization, as shown in Fig. (2). The HBM test method was in adherence to Telcordia TR-NWT-000870. For simplicity, the ESD pulse was applied to the device one time. A small group of laser devices was subjected to 10 ESD pulses for comparison. To determine the ESD damage threshold, lasers were subjected to low level of ESD stress starting from $0.5 \mathrm{kV}$ and increasing subsequently with a $0.25 \mathrm{kV}$ increment until failure. Since reverse polarity has been shown to be more sensitive than the forward polarity, reverse ESD threshold was measured in the study. Light $v s$ current (LI) was measured before and after each ESD stress to determine the ESD failure threshold. A threshold current change of over $3 \mathrm{~mA}$ was taken as the criterion for ESD failure [12]. The $3 \mathrm{~mA}$ failure criterion was adopted in this study in order to detect the early stage of ESD damage, while not being affected by the measurement noise that was about $0.5 \mathrm{~mA}$.

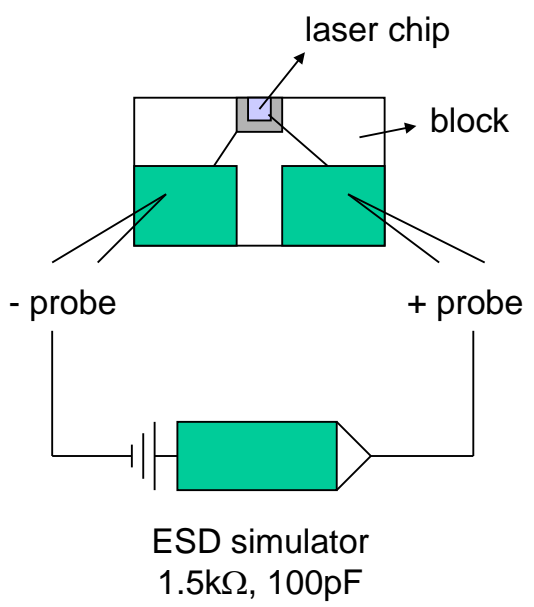

Fig. (2). Schematics the experimental setup of ESD stress.

\section{RESULTS AND DISCUSSION}

\section{(a) ESD Degradation Characteristics}

As shown in the previous paper [7], two types of ESD degradation were observed: hard and soft degradations. The hard degradation showed a sudden jump in the threshold current during the ESD voltage ramp, as shown in Fig. (3) where BH lasers with $360 \mu \mathrm{m}$ cavity and $6.0 \mu \mathrm{m}$ contact width were studied. When the hard ESD degradation occurred, the threshold current (Ith) exhibited a sudden jump from about $7 \mathrm{~mA}$ to $30-50 \mathrm{~mA}$ or above (Fig. 3a). The slope efficiency exhibited a precipitous drop from about 0.4 $\mathrm{mW} / \mathrm{mA}$ to about 0 , indicating that the laser has become dead or nearly dead (Fig. 3b). The dead laser was confirmed by direct probing on the metal stripe. The IV and turn-on voltage were normal, while the optical power was nearly zero. The soft breakdown was characterized by a gradual increase in the threshold current as a function of ESD voltage, as shown in Fig. (4), where BH laser with $360 \mu$ m cavity and $6.0 \mu \mathrm{m}$ contact width were studied. As a result of ESD damage, lasers exhibited increases in the threshold current (Fig. 4a) and corresponding decrease in the slope efficiencies (Fig. 4b). Fig. (5a) shows the LI examples of hard degradation from samples\#h-3 and \#h-4. The lasers of hard degradation became dead (\#h-3) or nearly dead (\#h-4) at ESD damage threshold. On the other hand, the LI exhibited more gradual change in the case of soft degradation (Fig. 5b).

Ueda [13] observed "rapid" and "gradual" degradations similar to the aforementioned hard and soft degradations. The gradual degradation was attributed to the formation of point defect clusters, while the rapid degradation was related to the recombination-enhanced dislocation climb or glide. 

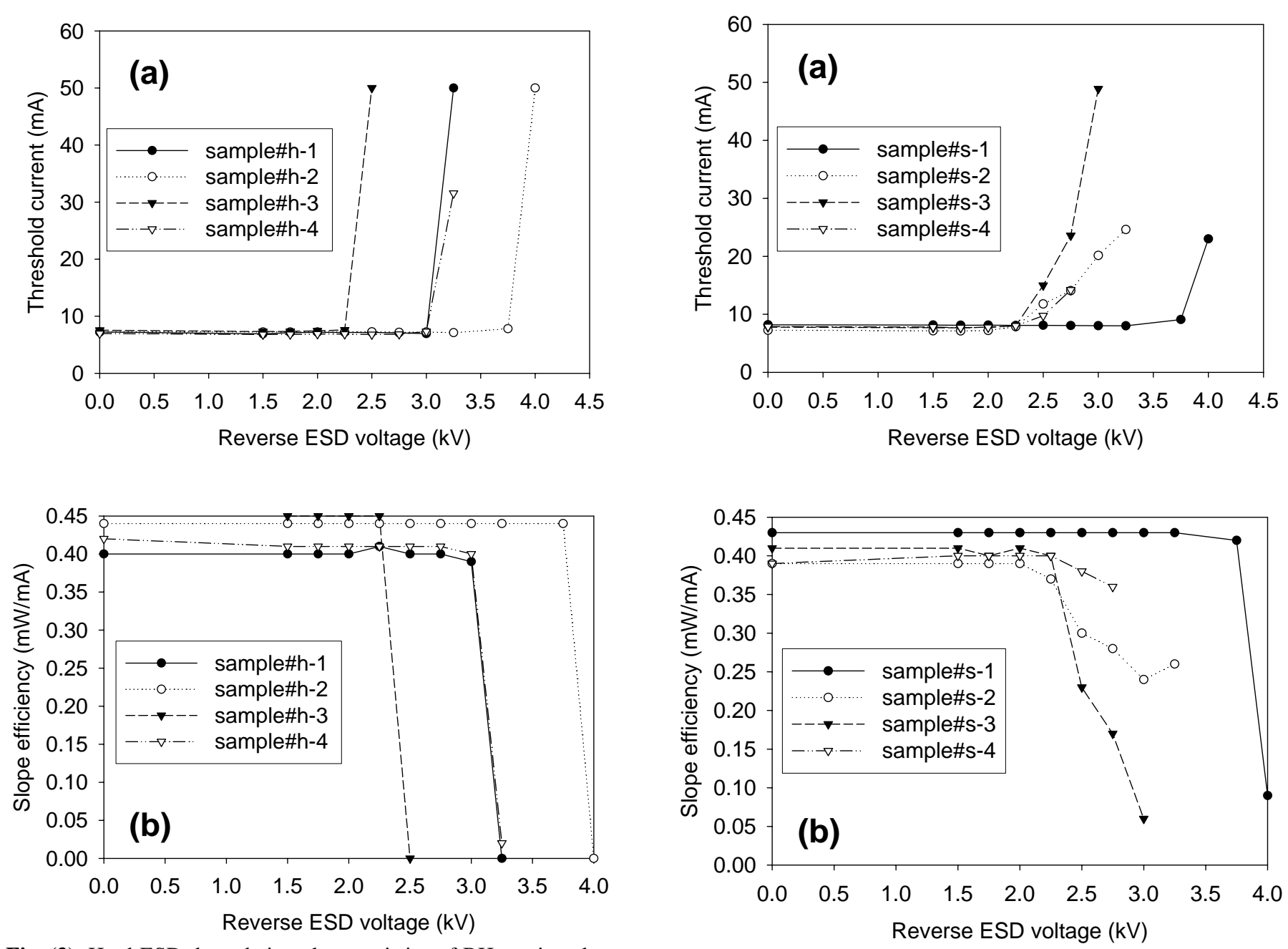

Fig. (3). Hard ESD degradation characteristics of BH semiconductor lasers shown in (a) Threshold current and (b) slope efficiency. Symbols represent different lasers.

Although the defects were studied and reviewed by transmission electron microscopy (TEM), the correlation between ESD degradation and size effect was not reported. In the next sections, we will discuss the detailed results of size effect, fabrication processes (laser structure and dielectric property) and ESD pulses.

\section{(b) Size Effect}

Fig. (6) shows the occurrence frequency of hard degradation as a function of cavity length. The sample size of each laser cavity was 15 . The contact width of the BH lasers was about $6.0 \mu \mathrm{m}$. The occurrence frequency was calculated based on the number of hard failure divided by the sample size. The $\mathrm{R}^{2}$ was the correlation factor of linear fitting where $\mathrm{R}^{2}=1$ represented perfect correlation and $\mathrm{R}^{2}=0$ represented no correlation. The occurrence frequency of hard ESD degradation decreased with increasing cavity length. For example, $80 \%$ of the $250 \mu \mathrm{m}$ lasers failed by hard degradation, while only $10 \%$ of the $550 \mu \mathrm{m}$ lasers failed by hard degradation. No hard degradation occurred for the $750 \mu \mathrm{m}$ lasers. As discussed in the previous paper [6], the dependence of cavity length might be attributed to the difference in energy flux. For the shorter laser cavity, the energy flux injected into the laser cavity was greater. Hence, the possibility for a

Fig. (4). Soft ESD degradation characteristics of BH semiconductor lasers shown in (a) Threshold current and (b) slope efficiency. Symbols represent different lasers.

catastrophic degradation (i.e. hard degradation) was more likely to increase. For the longer cavity, the energy flux was less, therefore the laser tended to degrade slowly rather than become suddenly dead.

\section{(c) Cavity Length Dependence}

Fig. (7) shows the reverse ESD threshold of BH lasers as a function of cavity length. The contact width of the $\mathrm{BH}$ laser is about $6.0 \mu \mathrm{m}$. The correlation plot indicated that the reverse ESD threshold increases linearly with the cavity length. The error bars represent the standard deviation. As the laser cavity increases from $250 \mu \mathrm{m}$ to $750 \mu \mathrm{m}$, the median value of reverse ESD thresholds increases linearly from $2.25 \mathrm{kV}$ to $6.75 \mathrm{kV}$.

There is a performance tradeoff between short and long laser cavities. The long cavity favors high-power operation, while the short cavity favors the speed [14]. Care needs to be taken to incorporate the ESD robustness when designing the high-speed laser.

\section{(d) Contact Width Dependence}

Fig. (8) shows the reverse ESD threshold of BH lasers as a function of contact width. The cavity length of the $\mathrm{BH}$ la- 

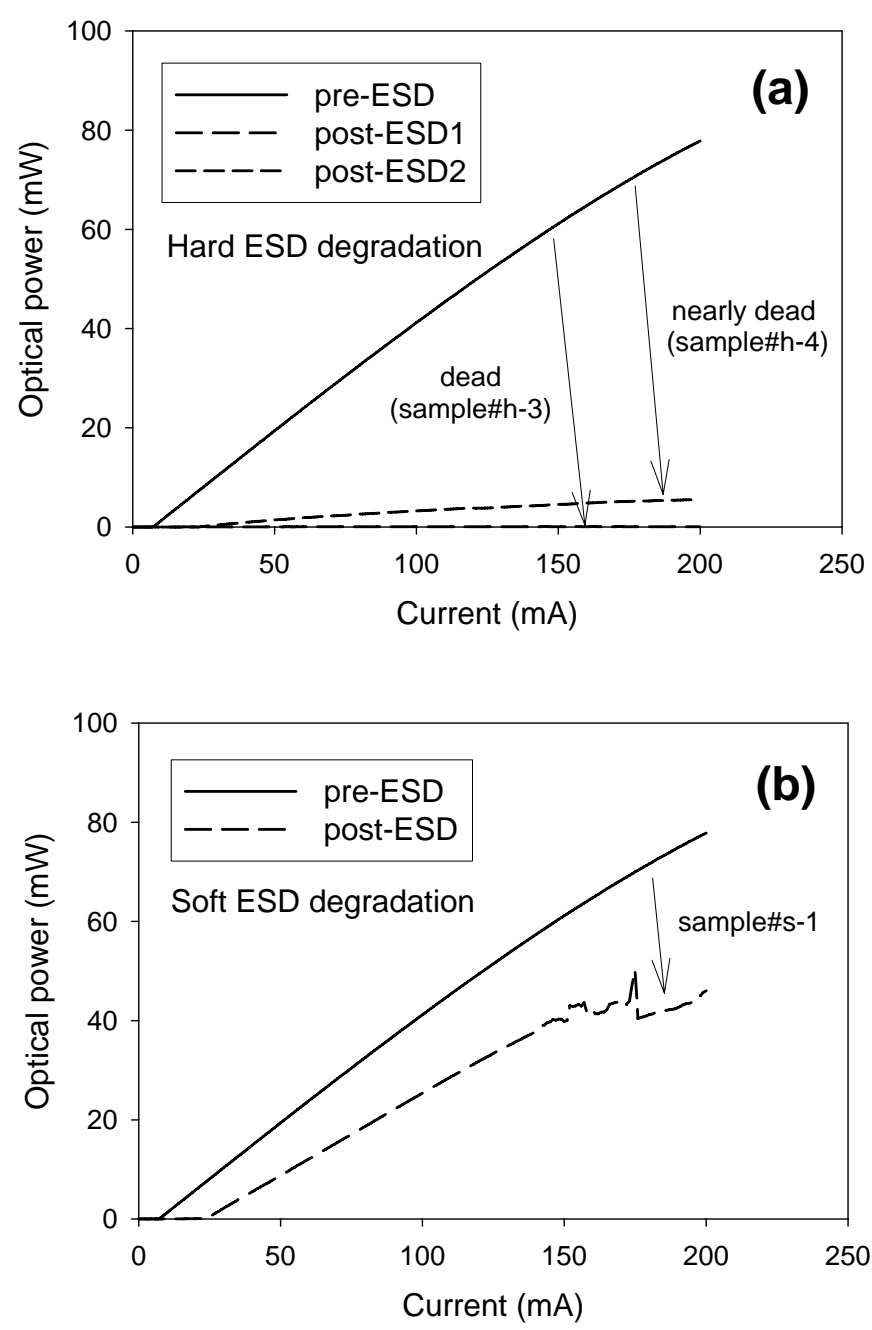

Fig. (5). LI curves of (a) hard (sample\#h-3 and \#h-4) and (b) soft degradation (sample\#s-1). Lasers with cavity length of $360 \mathrm{~mm}$ and contact width of $6 \mathrm{~mm}$ are used.

ser is $360 \mu \mathrm{m}$. The correlation plot indicates that the reverse ESD threshold increases linearly with the cavity length. As the contact width increases from $5.3 \mu \mathrm{m}$ to $8.1 \mu \mathrm{m}$, the median value of reverse ESD thresholds increases from $2.31 \mathrm{kV}$ to $4.05 \mathrm{kV}$. Based on the linear scaling from the fitting in Fig. (8), the ESD threshold of the $8.1 \mu \mathrm{m}$ contact is estimated to be $3.51 \mathrm{kV}$, lower than the observed value of $4.05 \mathrm{kV}$. Such deviation could be attributed to the dielectric undercut and current crowding.

Small contact width is typically employed to improve the current confinement and reduce the parasitic capacitance [15]. However, the ESD sensitivity is likely to be higher based on the data in Fig. (8). Therefore, the laser designer may need to carefully evaluate the ESD requirement and incorporate other design-in ESD reliability parameters as necessary.

\section{(e) Influence of Dielectric}

Fig. (9) shows the cumulative distribution plot of reverse ESD thresholds of lasers with thin and thick dielectric isolation layers where the cavity length of the lasers is $360 \mu \mathrm{m}$. The thick dielectric stack consists of $170 \mathrm{~nm} \mathrm{Si}_{3} \mathrm{~N}_{\mathrm{x}} / 400 \mathrm{~nm}$

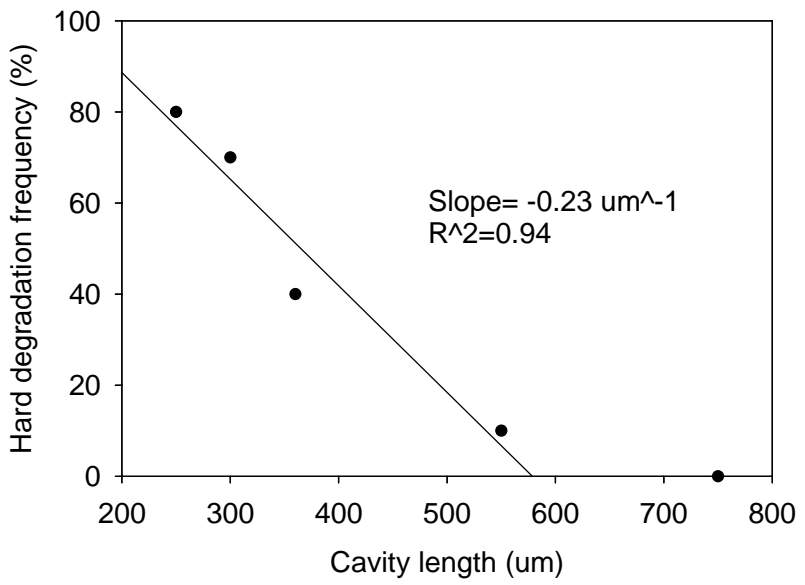

Fig. (6). Frequency of hard ESD breakdown as a function of cavity length. $\mathrm{R}^{2}$ is the correlation factor where 1.00 represents perfect correlation while 0 represents no correlation.

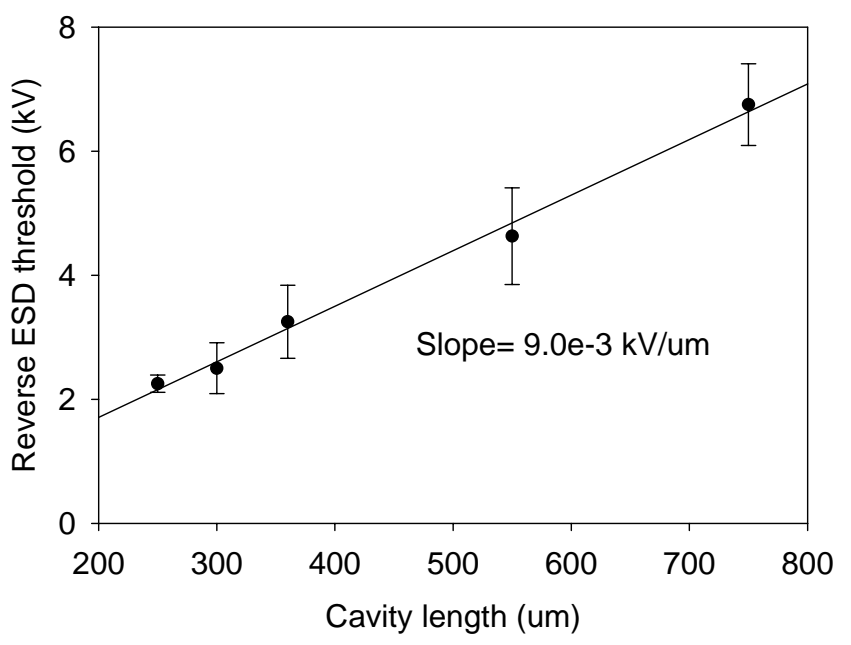

Fig. (7). Reverse ESD threshold of BH lasers as a function of cavity length. The contact width was about $6.0 \mathrm{~mm}$.

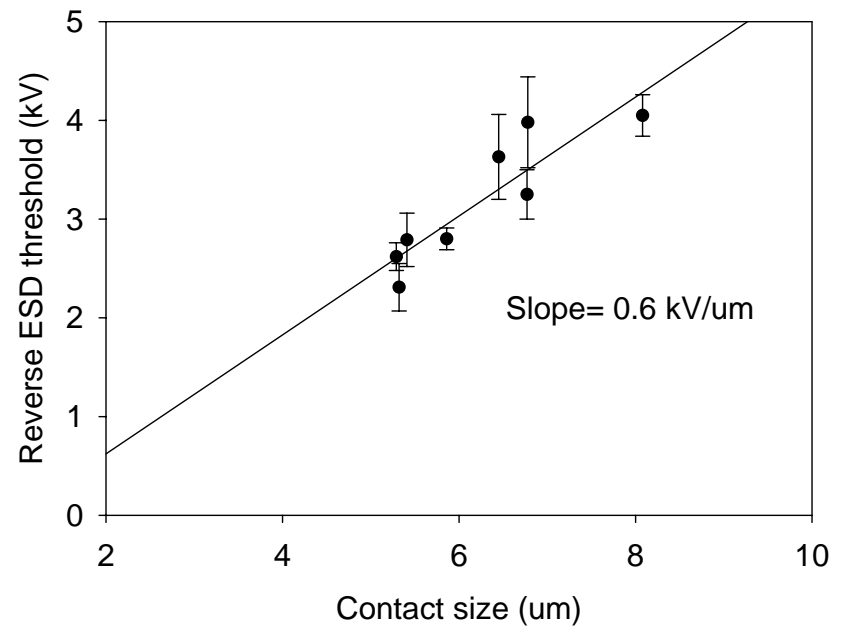

Fig. (8). Reverse ESD threshold of BH lasers as a function of contact width. The cavity length was $360 \mathrm{~mm}$.

$\mathrm{SiO}_{2} / 50 \mathrm{~nm} \mathrm{Si}_{3} \mathrm{~N}_{\mathrm{x}}$, while the thin dielectric stack consists of $100 \mathrm{~nm} \mathrm{Si}_{3} \mathrm{~N}_{\mathrm{x}} / 150 \mathrm{~nm} \mathrm{SiO}_{2}$. In the failure distribution plot, each data point represents one laser device. A total of 17-25 
lasers are tested in each group. The ESD damage threshold is determined by the ESD stress voltage at which laser exhibits threshold degradation of $>3 \mathrm{~mA}$. The ESD damage thresholds are sorted out in ascending order to construct the cumulative failure distribution. The median and sigma are estimated using lognormal distribution function. The median values of the reverse ESD thresholds for thin and thick dielectric layers are $3.16 \mathrm{kV}$ and $2.89 \mathrm{kV}$, respectively. In addition, an early failure "tail" at $1.5-2.25 \mathrm{kV}$ occurs in the thick dielectric group. We attribute the higher ESD threshold and tighter distribution of the thin dielectric to the improved metal conformity. As shown in Figs. (10a, b), the metal coverage on the top of the laser structure with the thin dielectric is conformal, while the metal step coverage is poor at the edges of the contact window for the thick dielectric. The poor step coverage is likely a source of local heating, leading to early ESD degradation.

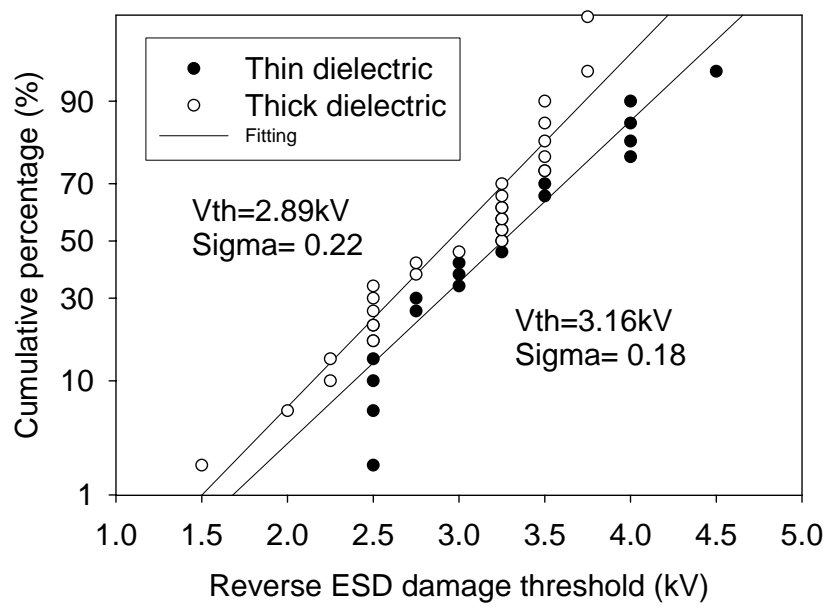

Fig. (9). Failure distributions of ESD thresholds of BH lasers with thin and thick dielectric layers. Laser devices were subject to reverse polarity of ESD stress.

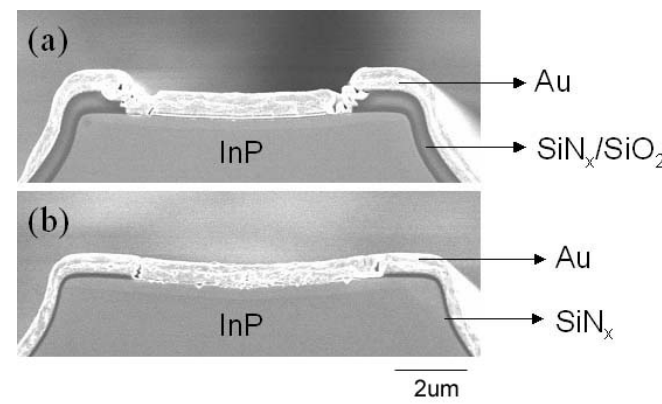

Fig. (10). SEM cross-sections of BH lasers with (a) thick and (b) thin dielectric layers. The dielectric thickness resulted in different metal film conformity.

Thick dielectric is typically used to improve the device parasitic due to the reduction in capacitance [15]. However, the tradeoff in ESD performance may occur as a result of current crowding associated with the morphological nonuniformity. Consideration in ESD performance is important during the processing design phase.

\section{(f) Number of ESD Pulses}

Fig. (11) shows the reverse ESD threshold as a function of number of pulses where $300 \mu \mathrm{m} \mathrm{BH}$ lasers are tested. The data showed that the ESD thresholds of the lasers subjected to $10 \mathrm{ESD}$ pulses are lower than those subjected to $1 \mathrm{ESD}$ pulse. The median ESD thresholds for the lasers subjected to 10 ESD pulses and 1 ESD pulse are $1.8 \mathrm{kV}$ and $2.2 \mathrm{kV}$, respectively. For each ESD pulse condition, ten lasers from the two wafers are sampled for testing. To minimize other variables, ten lasers are randomly selected from each wafer and split into two groups. One half is tested with 1 ESD pulse (pulse $\mathrm{x} 1$ ), and the other is tested with 10 ESD pulses (pulse $\mathrm{x} 10)$. Both wafers show consistently lower ESD thresholds for the $10 \mathrm{ESD}$ pulse condition.

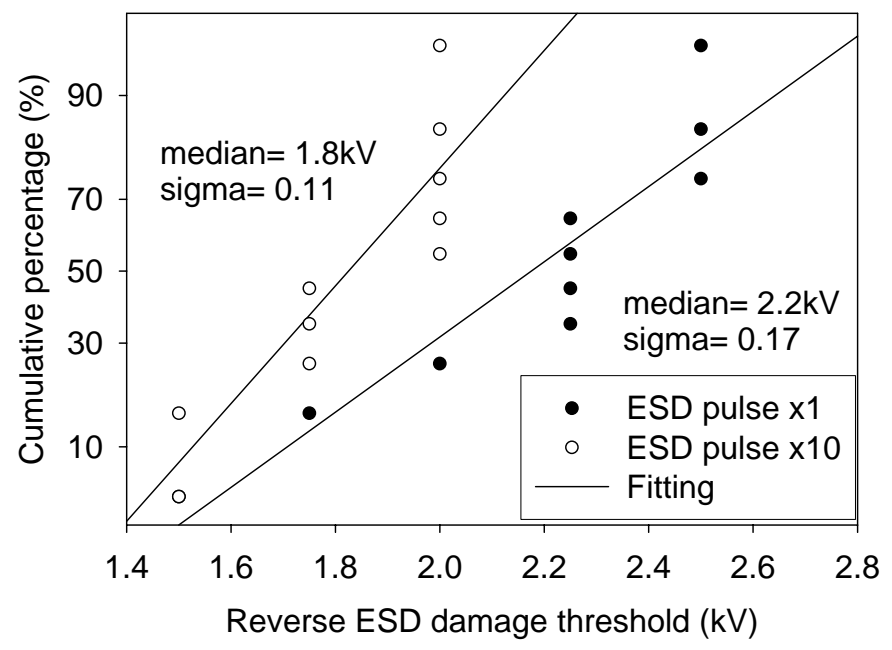

Fig. (11). ESD cumulative failure distributions of lasers subjected to 1 ESD pulse and 10 ESD pulses.

Based on the finding of the ESD dependence on stress frequency, it is important to consider the testing methodology when comparing the ESD data between vendors. For a good baseline comparison, knowledge of the laser package such as AlN submount or butterfly package may be important [16]. For example, the RF circuit in the package may interact and respond differently with the human-body-model (HBM) ESD stress and hence affect the ESD results.

\section{(g) BH vs RWG Lasers}

Fig. (12) compares the reverse ESD threshold of BH and ridge waveguide (RWG) lasers. The data of long cavity RWG lasers are not available since RWG is typically designed for high-speed applications that require short cavities. For the same cavity length, the RWG lasers exhibit lower ESD threshold. We attribute the lower threshold to the narrow ridge width. The width dependence also supports the data shown in Fig. (8) where the reverse ESD threshold increases with the contact width. The ESD threshold does not seem to scale linearly with the contact width. For example, the ESD thresholds of the $300 \mu \mathrm{m} \mathrm{BH}$ and RWG lasers are $2.5 \mathrm{kV}$ and $1.65 \mathrm{kV}$, respectively. The contact widths of the $\mathrm{BH}$ and $\mathrm{RWG}$ lasers are about $5.5 \mu \mathrm{m}$ and $2.0 \mu \mathrm{m}$, respectively. The ratio of ESD threshold of $\mathrm{BH} v s \mathrm{RWG}$ is 1.54 , while the ratio of contact width is 2.75 . The non-linear relationship is likely due to current crowding and other processing variables. It is noted that the ridge laser exhibited higher ESD threshold than the expected value based on linear width scaling. The higher-thanexpected ESD threshold of the ridge laser is likely related to less current crowding. Detailed simulation may be needed to further quantity the effect of each factor. 


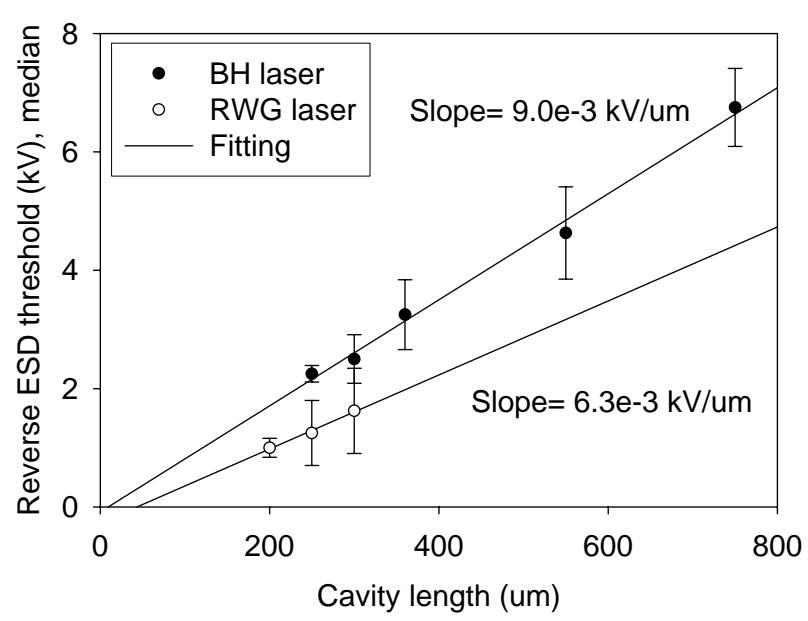

Fig. (12). Comparison of ESD thresholds of BH and RWG lasers.

Ridge waveguide design is employed for low cost applications where the current confinement is achieved by ridge etch without involving any epitaxial regrowth. However, the data in Fig. (12) suggests that there may be an increase in ESD sensitivity for the ridge structure as a tradeoff.

\section{CONCLUSION}

We have extensively studied the size effect on ESD threshold and degradation behavior of the BH DFB InP lasers using the HBM model. We found two types of ESD breakdown behaviors: soft and hard degradations. The soft degradation showed a gradual threshold current degradation, while the hard degradation showed a sudden jump in the threshold current. We showed that the frequency of the hard degradation decreased with increasing cavity length: $80 \%$ if the $250 \mu \mathrm{m}$ lasers exhibited hard degradation, while none of the $750 \mu \mathrm{m}$ lasers exhibited such behavior. The reverse ESD damage threshold increased with increasing cavity length and contact width. The size effect could be explained by the energy flux. For the larger chip, the energy flux was smaller, so the laser could sustain a higher ESD stress. The ESD threshold increased following linear scaling with the cavity length, but not with the contact width. For the latter, the non-linear deviation could result from dielectric undercut and current crowding.

Besides the size effect, we also showed that dielectric layer, ESD pulse frequency and laser structure could be influential in ESD performance. For the thin dielectric, the metal coverage was more uniform, thereby the heat caused by ESD stress was more evenly distributed. For the thicker dielectric, the metal step coverage was poor. The poor step coverage could potentially cause local heating, leading to early ESD failure. For the pulsing frequency, the ESD threshold of the $300 \mu \mathrm{m}$ was reduced from $2.2 \mathrm{kV}$ for $1 \mathrm{ESD}$ pulse to $1.8 \mathrm{kV}$ for $10 \mathrm{ESD}$ pulses. For the laser structure, comparison of $\mathrm{BH}$ and RWG lasers showed that RWG lasers typically showed lower reverse ESD thresholds than the $\mathrm{BH}$ for a given cavity length. The reduction in the ESD thresholds was attributed to the narrower contact width.

\section{ACKNOWLEDGEMENTS}

The authors would like to thank Eric Isip for supply of the ESD simulator, Cherrena Pruitt for chip delivery and Chris Larocca for reviewing the paper.

\section{REFERENCES}

[1] TR-NWT-000870, Electrostatic discharge control in the manufacture of telecommunications equipment, Bellcore, Telcordia, Morristown, NJ, 1991.

[2] JEDEC Standard JESD22-A114D, Electrostatic discharge (ESD) sensitivity testing human body model (HBM), JEDEC Standard Arlington, VA, 2006

[3] Twu Y, Cheng LS, Chu SNG, Nash FR, Wang KW, Parayanthal KW. Semiconductor laser damage due to human-body-model electrostatic discharge. J Appl Phys 1993; 74(3): 1510-20.

[4] Jeong J, Park KH, Park HM. Wavelength shifts of 1.5- $\mu \mathrm{m}$ DFB lasers due to human-body-model electrostatic discharge followed by accelerated aging experiments. J Lightwave Technol 1995; 13(2): 186-90.

[5] DeChiaro LF, Unger BA. Degradation in InGaAsP semiconductor lasers resulting from Human body model ESD. J Electrostat 1993; 29: $227-50$.

[6] Wallon J, Terol G, Bauduin B, Devoldere P. Sensitivity to electrostatic discharges of "low-cost" $1.3 \mu \mathrm{m}$ laser diodes: a comparative study. Mater Sci Eng 1994; B28: 314-18.

[7] Huang JS, Olson T, Isip E. Human-body-model electrostatic discharge and electrical overstress studies of buried heterostructure distributed feedback lasers. IEEE Tran Dev Mater Reliab 2008; 7(3): 453-61

[8] Huang JS. ESD breakdown characteristics of buried heterostructure semiconductor lasers, Compound Semiconductor Manufacturing Expo 2005. Institute of Physics, Palm Springs, CA, Oct. 30- Nov. 3, 2005, pp. 63-5.

[9] Neitzert HC, Piccirillo A. Sensitivity of multimode bidirectional optoelectronic modules to electrostatic discharges. Microelectron Reliab 1999; 39: 1863-71.

[10] Su YK, Chang SJ, Wei SC, Chen SM, Li WL. ESD Engineering of nitride-based LEDs. IEEE Tran Device Mater Reliab 2005; 5(2): 277-81.

[11] DeChiaro LF, Sandroff CJ. Improvement in electrostatic discharge performance of InGaAsP semiconductor lasers by facet passivation. IEEE Trans Electron Device 1992; 39(3): 561-5.

[12] Sim SP, Robertson MJ, Plumb RG. Catastrophic and latent damage in GaAlAs lasers caused by electrical transients. J Appl Phys 1984; 55(11): 3950-5.

[13] Ueda O. Degradation of III-V opto-electronic devices. J Electrochem Soc 1988; 135(1): 11-22.

[14] Chen TR, Ungar J, Yeh XL, Bar-Chaim N. Very large bandwidth strained MQW DFB laser at 1.3mm. IEEE Photon Tech Lett 1995; 7(5): 458-60

[15] Lu H, Blaauw C, Benyon B, Li GP, Makino T. High-power and high-speed performance of $1.3-\mu \mathrm{m}$ strained MQW gain-coupled DFB lasers. IEEE J Select Topic Quantum Electron 1995; 1(2): 375-81.

[16] Huang JS, Miao R, Lu H, Wang C, Helms C, Demirci H. Theoretical and experimental thermal analysis of InP ridge lasers on submounts and TO packages. IEEE Tran Dev Mater Reliab 2007; 7(2): 363-8. 